Methods An online survey was designed based on a large-scale in depth focus discussion study among STI experts and professionals and distributed via email to current IUSTI members. Conditional logistical regression modelling will be used for data analysis. We present preliminary data here.

Results To date, 142 subjects took the online survey with 123 completing it: $44 \%(n=63)$ male and $56 \%(n=79)$ female. Most subjects were from Oceana (35\%) followed by Europe (18\%), America $(18 \%)$, Africa $(15 \%)$ and Asia (14\%). The majority $(59 \%)$ of participants were from developed countries. Unreliability (17\%) was the greatest barrier for use of POCTs, followed by being laboratorydriven $(15 \%)$ and time- frame (13\%). Perceptions of STI POCT differed significantly between developing and developed country participants. The majority (85\%) of participants from developing countries thought test cost was more important versus $67 \%$ from developed countries $(p<0.05)$. Participants from developing countries ranked early HIV seroconversion as top priority for new STI POCT while those from developed countries chose chlamydia. Only $24 \%$ from developing countries preferred to prioritise the development of chlamydia POCT as compared to $57 \%$ from developed countries.(p value?) In addition, the majority (53\%) of participants from developed countries preferred a POCT with higher sensitivity but longer turn-around-time and much more expensive but only $28 \%$ from developing countries preferred this POCT ( $p<0.05)$.

Conclusion One STI POCT may not fit all. Industry should consider country identified needs in development of future acceptable, usable STI POCT.

\section{P5.084 MULTIPLEX CAPABILITY OF A FULLY-INTEGRATED, LOW- COST, ULTRA-RAPID PCR DEVICE WITH POINT-OF-CARE APPLICATIONS}

doi:10.1136/sextrans-2013-051184.1128

D Pearce, B Marsh. Atlas, Trowbridge, UK

Background We have developed a novel Point-of-Care molecular assay system, io $^{\mathrm{TM}}$, comprising an assay-specific Cartridge and Reader. With a turnaround time of just 30 minutes the System has an initial focus on rapidly detecting sexually-transmitted infections (STIs). The System has been developed to run tests that simultaneously detect Chlamydia trachomatis (CT) and Neisseria gonorrhoeae (NG), each run with an internal control (IC). The assays utilise a novel electrochemical method that demonstrates low copy number amplification and detection. Here, we have developed a CT/NG/IC triplex assay where each target analyte is co-amplified prior to being differentially detected.

Methods The assays were run using prototype PCR Cartridges in conjunction with an ultra-rapid thermocycler. All reagents necessary to perform the assay were deposited into the Cartridge. A sample was added to the Cartridge, DNA extracted, and the resulting eluate reconstituted dried amplification reagents. Amplified targets were detected using electrochemically-labelled target-specific probes and a double-stranded DNA-specific nuclease to release the electrochemical labels. Released labels were detected by applying a voltage to a screen-printed carbon electrode. Measurable current at specific oxidation potentials indicated the presence of targets in the sample. Results Initial analytical sensitivity of the triplex CT/NG/IC assay was evaluated by testing each target analyte in combination with various concentrations of the other two (including negative controls). Electrochemical detection demonstrated clear differentiation between peaks generated by each cleaved label and showed a limit of detection of ten genome copies. Negative samples showed no significant peaks.

Conclusions The results showed that reliable, differentiated detection of three targets in a single sample was possible across a wide range of concentrations of the three targets. While demonstrated here for three analytes, the Atlas high-multiplex technology will allow expansion of the Atlas io ${ }^{\mathrm{TM}}$ test menu to detect multiple STIs in a single sample.

\section{P5.085 TREPONEMA PALLIDUM ANTIBODIES DETECTION BY A POINT-OF-CARE TEST AND RPR AND TPHA TESTS IN MSM ATTENDING A COMMUNITY BASED HIV ANONYMOUS CENTER - CHECKPOINT LX}

doi:10.1136/sextrans-2013-051184.1129

1,2R Castro, ${ }^{1} \hat{A}$ Lopes, ${ }^{3} \mathrm{R}$ Fuertes, ${ }^{3} \mathrm{H}$ Machado, ${ }^{3} \mathrm{M}$ Rocha, ${ }^{3} \mathrm{R}$ Jordão, ${ }^{3} \mathrm{~J}$ Brito, ${ }^{3} \mathrm{~J}$ Esteves, ${ }^{3} \mathrm{M}$ J Campos, 'F Pereira. 'Instituto de Higiene e Medicina tropical, Lisboa, Portugal; ${ }^{2}$ Centro de Recursos Microbiológicos-CREM, Lisboa, Portugal, ${ }^{3}$ CheckpointLX, Lisboa, Portugal

Background When available, point-of-care tests are very useful in the screening of STIs. They have affordable prices, need minimal equipment and training, results are immediately available, allowing treatment with no delay.

Objectives We describe the prevalence of syphilis in an MSM population attending an HIV Anonymous Testing Center (ATC), who stated that they were never infected with Treponema pallidum, as evaluated with a rapid test. Positive results were confirmed with the RPR and TPHA tests.

Materials and Methods Nine hundred and forty four individuals attending the HIV ATC were tested with the Determine Syphilis TP test. Those who were found to have reactive results had blood taken for confirmation with RPR and TPHA tests.

Results The rapid test was reactive in 44 of the 944 (4.7\%) individuals. Samples were further tested with the RPR and the TPHA tests; 34 showed to have antibodies against T. pallidum in both tests, although one sample was reactive only at the 1:2 dilution in the RPR and its TPHA titer was 1:640. Six samples were only reactive for the TPHA test, while four were non reactive in both tests. The FTA-ABS was performed in these four samples and it was non reactive.

Discussion and conclusion: In accordance with the results of the rapid test, the percentage of reactive samples was $4.7 \%$ (44/944). However, when confirmatory tests were performed in the samples received in the laboratory, the percentage of reactive samples decreased to $4.2 \%$ (40/944). Furthermore, in six of these samples only the TPHA was reactive, meaning that these patients probably had a treated past infection, which was not detected as such by the rapid test.

In conclusion, the Determine Syphilis TP test seems to be useful as a screening test for syphilis, although it does not differentiate between treated and active syphilis.

\section{P5.086 DIAGNOSIS OF EXTRA-GENITAL CHLAMYDIA AND/OR GONORRHOEA INFECTIONS BY VERSANT CT/GC DNA 1.0}

doi:10.1136/sextrans-2013-051184.1130

'A Marangoni, ' $\mathrm{C}$ Foschi, ${ }^{1} \mathrm{P}$ Nardini, ${ }^{1} \mathrm{M}$ Compri, ${ }^{2} \mathrm{~A}$ D'Antuono, ${ }^{2} \mathrm{~S}$ Bellavista, ${ }^{2} \mathrm{~A}$ Filippini, ${ }^{3} \mathrm{M}$ Capretti, 'R Cevenini. 'University of Bologna, Microbiology, DIMES, Bologna, Italy; ${ }^{2}$ University of Bologna, Dermatology, DIMES, Bologna, Italy; ${ }^{3} S$. Orsola Hospital, Neonatology, Bologna, Italy

Objectives Nucleic acid amplification testing (NAAT) has become the preferred method to detect Chlamydia trachomatis (CT) and Neisseria gonorrhoeae (GC) infections. Anyway, no commercial test has been cleared so far for use with extra-genital swab samples.

In this study Versant CT/GC DNA 1.0 (Siemens) performances have been evaluated by testing ocular, rectal or pharyngeal secretions collected by Siemens collection devices.

Methods Study group. A prospective study was performed with 7 newborns with conjunctivitis, and 183 subjects attending the STD Outpatients Clinic of St. Orsola Hospital, Bologna. The latter ones were enrolled because having unsafe receptive anal and/or pharyngeal sex intercourses. 